\title{
Pour un contexte épistémologique du présent. Ou comment se libérer des apories d'une postmodernité généreuse mais fatiguée
}

\author{
Noël Cordonier et Sonya Florey \\ Haute École Pédagogique du Canton de Vaud, Suisse
}

\section{Résumé}

Cet article se propose d'analyser une expérience scolaire qui a permis aux élèves d'une classe primaire de créer un imagier plurilingue, en sollicitant l'aide de leurs parents francophones ou allophones et de l'intégrer dans des tâches d'apprentissage. Notre analyse emprunte à divers référents et disciplines et nous soumettons ainsi au lecteur un cadre de pensée qui recourt d'abord à la notion de postmodernité et notamment au célèbre débat entre Lyotard et Habermas, puis au concept d'identité telle qu'elle est traitée dans le roman Rosie Carpe de Marie Ndiaye et dans l'ouvrage de Pierre Bayard Et si les auvres changeaient d'auteur?, et enfin au modèle proposé par Nicolas Bourriaud, dans son essai Radicant, qui ajoute à la désormais connue postmodernité, un cadre théorique supplémentaire, l'altermodernité. On éclairera ainsi l'imagier plurilingue, qui sollicite un ensemble de 16 langues (albanais, allemand, anglais, arabe moderne, espagnol, italien, kurde kurmandji, kurde sorani, mooré, polonais, portugais, serbe, somali, tagalog, thaï, turc) par la notion de « trajet ».

\section{Mots-clés}

Plurilinguisme, postmodernité, altermodernité, identité, imagier.

\begin{abstract}
This paper analyses the creation of a multilingual book by pupils and their parents, coming originally from the francophone or the allophone community. The project involves 16 languages present in the classroom (Albanian, German, English, modern Arabic, Spanish, Italian, Kurdish Kurmandji, Kurdish Sorani, Moré, Polish, Portuguese, Serbian, Somali, Tagalog, Thai, Turkish). The theoretical framework we use was built from various sources : first, we introduce the notion of postmodernity, with the famous debate between Lyotard and Habermas, then the concept of identity as set out in the novel Rosie Carpe of Marie Ndiaye and in the essay Et si les æuvres changeaient d'auteur? of Pierre Bayard, finally the notion of « altermodernity », such as defined by Nicolas Bourriaud in his essay Radicant.
\end{abstract}

\section{Keywords}

Multilingualism, postmodernity, altermodernity, identity, multilingual book. 


\section{Introduction}

Depuis une vingtaine d'années, les recherches en sciences de l'éducation et en didactique ont pratiquement renoncé aux réflexions théoriques ou épistémologiques, ainsi que suffit à le montrer la consultation des revues professionnelles ou celle des Actes de la plupart des colloques. Après qu'elles eurent adopté des techniques et des protocoles rigoureux, reposant sur des prises de données explicites, nos recherches ont appliqué un purisme méthodologique strictement calqué sur celui des sciences dites exactes, qui a écarté quasi toute considération spéculative, souvent soupçonnée de subjectivité et d'impressionnisme. De gratuité et d'inefficacité aussi.

Ce faisant, nos disciplines ont indubitablement amélioré leur ancrage dans la recherche internationale, essentiellement pragmatique et empirique. Mais avons-nous réellement " progressé »? Si, dans les sciences dites exactes, les savoirs et les connaissances sont le plus souvent cumulatifs (par exemple, on en sait chaque jour davantage sur le boson de Higgs), peut-on dire que les sciences humaines (sciences de l'éducation, philosophie, littérature...) progressent? Ne sommes-nous pas, en ces cas, perpétuellement et seulement en train d'adapter nos instruments et nos observations à la réalité humaine et sociale telle que le présent et le temps nous la font incessamment apparaître et varier? Aujourd'hui, commentons-nous l'être humain mieux qu'Aristote ou que Kant? Ne le disons-nous pas, simplement, autrement?

Peu sensible aux modes de l'heure et au monisme méthodologique, et en partie inspirée par les précédents travaux sur les didactiques contextualisées qui ont toujours su laisser vivre la réflexion (Blanchet, 2010; Castellotti et Moore, 2008; Robillard, 2008), la question de recherche qui a abouti au $1^{\text {er }}$ colloque international du CRREF (EA 4538) intitulé «Contextualisations didactiques : états des lieux, enjeux et perspectives » et organisé en novembre 2011 en Guadeloupe, a, au contraire, judicieusement sollicité, à côté des études empiriques rigoureuses, " des travaux présentant des cadres théoriques et méthodologiques spécifiques » (CRREF, 2011, Problématique du colloque) ${ }^{1}$ pour discuter, encore et toujours, pour ajuster, inlassablement, nos rapports à nos objets d'étude.

Notre article souhaite modestement répondre à cette ouverture et à cette offre, en se proposant d'interroger et de discuter l'idéologie qui semble actuellement nous procurer les concepts et les postulats majeurs pour penser l'élève contemporain dans son contexte. Si ce contexte comprend bien l'ensemble des «conditions historiques, géographiques, sociales, culturelles, professionnelles, religieuses, générationnelles, de genre, etc. qui constituent l'ancrage des phénomènes humains et sociaux dans une construction dialectique » (Blanchet et Chardenet, 2011 : 448), notre intention est d'ajouter à la liste ouverte des entrées celle qui nous paraît la plus large, l'épistémologie qui sous-tend la didactique du plurilinguisme. Selon nous, les manières d'envisager l'être humain en général, ou l'élève en particulier, dans son temps, son espace, son individualité, son sexe, son groupe, ses langues, sa culture, ses valeurs sont aussi déterminées par l'épistémologie générale, par la philosophie que notre époque a données à l'observateur et l'acteur que nous sommes, par ses croyances en ces domaines.

A ce niveau général, malgré notre manque de distance pour bien la nommer, malgré notre inévitable ethnocentrisme, c'est la postmodernité qui nous paraît être, aujourd'hui, la Weltanschauung ${ }^{2}$ régissant actuellement les sciences humaines européennes et américaines.

\footnotetext{
${ }^{1}$ Voir : http://contextualisations.sciencesconf.org/resource/page?id=3

2 «Weltanschauung» est à entendre ici comme une «conception du monde », telle que décrite par les philosophes romantiques allemands du $\mathrm{XIX}^{\mathrm{e}}$ siècle. En sciences humaines, ce concept est régulièrement associé à celui de
} 
Innombrables, diverses et concurrentes sont cependant les définitions de la postmodernité ou du postmodernisme $e^{3}$, lesquels sont commentés aussi bien par l'histoire de l'art, l'architecture, la littérature, la sociologie et la philosophie. En préférant ici le terme de postmodernité, qui relève de la sociologie historique et de la philosophie, à celui de postmodernisme, désignant le mouvement artistique qui, en architecture et urbanisme d'abord, s'est distancié du modernisme, nous nous recommanderons principalement de l'approche de Jean-François Lyotard (1979). Dans La condition postmoderne, qui était initialement un « Rapport sur le savoir » commandé par le gouvernement du Québec, la postmodernité, dans sa plus courte saisie, est ainsi circonscrite : « En simplifiant à l'extrême, on tient pour postmoderne l'incrédulité à l'égard des méta-récits » (Lyotard, 1979: 46), c'est-à-dire les récits d'« émancipation » et de « spéculation » qui œuvraient auparavant à la légitimation du savoir (Lyotard, $1979: 55-60)^{4}$.

La notion même de postmodernité suscite aujourd'hui encore le soupçon. A l'époque de la parution de La condition postmoderne, un débat célèbre avait opposé Jean-François Lyotard et Jürgen Habermas : Lyotard, comme évoqué ci-dessus, voyait dans la faillite des métarécits le signe d'un passage à une autre configuration de société, tandis que Habermas défendait l'idée d'une modernité inachevée.

Le philosophe allemand reconnaissait les limites, les dérives, voire les apories du projet moderne, et déplorait en particulier la spécialisation ainsi que la normativité qui atteint les " sphères de valeurs propres à la science, à la morale et à l'art " (Habermas, 1981: 311). Pourtant, en rétablissant les liens entre la culture moderne et la pratique vécue, il souhaitait renouer avec le projet de la modernité (Ibid: 315 ).

Lyotard, quant à lui, associait la postmodernité à l'opportunité d'un sévère réexamen de la pensée des Lumières : par exemple, « [les] artistes et [les] écrivains doivent [...] interroger les règles de l'art de peindre ou de raconter telles qu'ils les ont apprises et reçues des prédécesseurs " (Lyotard, 1982: 319). Malgré la profonde remise en question des modèles antérieurs ainsi que des pratiques artistiques, le philosophe ne rendait pas les enjeux modernes de facto caducs. Le postmoderne « fait assurément partie du moderne » (Lyotard, 1982 : 321) : la filiation n'était en aucun cas rompue - malgré ce que des lectures mal informées de Lyotard ont pu laisser croire.

Les deux philosophes s'entendaient néanmoins sur un point essentiel, la reconnaissance d'une mutation de la société occidentale, tout en s'opposant sur le sens de cette dernière. Égarement de l'esprit contemporain, infidélité à l'unité moderne entre science, morale et art d'une part, entre connaissance et pragmatique d'autre part, pour le défenseur de la modernité. Dépassement et accomplissement des anciennes valeurs, dynamique naturelle de l'évolution des idéologies, pour le partisan de la postmodernité.

\footnotetext{
« paradigme » ( $\pi \alpha \rho \alpha ́ \delta \varepsilon \imath \gamma \mu \alpha$ / paradeїgma, en grec ancien), permettant de considérer des faits historiques, sociaux, politiques, culturels dans un contexte idéologique spécifique.

${ }^{3}$ Notre intention avouée étant précisément d'interroger les habitudes de pensée des enseignants et didacticiens que nous sommes et de tenter de les changer un tant soit peu, c'est un choix délibéré de notre part de ne pas convoquer ici les philosophes et les sociologues que l'on appelle le plus souvent pour commenter l'École moderne et postmoderne, à commencer par Hannah Arendt.

${ }^{4} \mathrm{Si}$ la postmodernité est liée aux crises de la représentation de l'homme, on peut la faire commencer au XIX ${ }^{\mathrm{e}}$, mais le scepticisme et le relativisme généralisés qui la caractérisent sont plus exactement des conséquences des guerres mondiales, du désenchantement religieux et de la fin du positivisme scientifique (à distinguer du pragmatisme actuel et de la technologie). De ce point de vue, par commodité, on datera la postmodernité de la réflexion de Lyotard, qui a coïncidé avec la fin des «Trente glorieuses», soit des années 1975-1980.
} 
Lyotard (1985) lui-même, quelques années après la publication de son ouvrage séminal, demeurait conscient du chemin qui restait à parcourir en vue de forger, non seulement une compréhension convaincante de la fin du $\mathrm{XX}^{\mathrm{e}}$ siècle, mais aussi un consensus à l'égard du concept : «Personne n'est encore capable de définir cette rupture postmoderne dans un sens qui ne soit pas lamentable et éclectique (commentaires d'art, architecture...). Nous sommes convaincus qu'elle va durer des décennies. Elle est inévitable. La tâche devant nous est d'essayer de fournir une légitimité pour la société à venir » (Lyotard, 1985 : 9).

Ainsi, historiquement, cette déflation générale, ce désenchantement, ce scepticisme à l'égard de la Transcendance et de la Raison ont été illustrés, avant et surtout après Lyotard, par bien des philosophes et des sociologues (Campbell, Rorty, Lipovetsky, Hargreaves, Bauman, Kaufmann, Dufour, Maffesoli, Virilio, etc.), qui ont collaboré à repérer et à recenser les effets de la postmodernité. Pour d'autres, la postmodernité est suspicieuse, tel Latour (1991), qui plaide pour un concept de «non-modernité ». Epistémologiquement, nous nous situons à la suite de ceux qui reconnaissent une rupture de société et qui la nomment "postmoderne", et modestement, nous souhaitons évaluer la portée du paradigme postmoderne à l'échelle de l'École, et plus précisément à celle de la didactique du plurilinguisme.

Avant d'en venir à l'École, énumérons les principales caractéristiques qui découlent de la conception ici retenue de la postmodernité :

1. Le relativisme généralisé (incertitudes morales, religieuses, et même scientifiques),

2. Le primat du présent (et, par corollaire, un éloignement du passé et un affaiblissement de la transmission),

3. Un individualisme hédoniste et/ou inquiet, ainsi que la quête identitaire de tout sujet, narcissique ou anxieuse, dans un monde aujourd'hui dérégulé,

4. Une conception multiple, éclatée, problématique de l'appartenance,

5. Une participation du sujet, plus ou moins soumise, plus ou moins lucide, à l'économie globalisée,

6. Une mobilité identitaire à la fois revendiquée (l'individu multiple et fragmenté) et imposée par le néolibéralisme économique et par la communication généralisée.

Selon nous, l'essentiel de ce qui est impliqué dans l'étude des contextualisations didactiques, à savoir « la prise en compte de la diversité culturelle des contextes plurilingues », « le processus de contact et de métissage des savoirs locaux et universels », et plus forfaitairement encore, « les relations au local et à l'universel » (CRREF, 2011, appel à communication) $)^{5}$ ne saurait ignorer ce cadre épistémologique englobant, actuellement représenté au mieux par la postmodernité.

Toutefois, si nous posons ce postulat au départ de notre réflexion, c'est moins pour nous y appuyer que pour tester certaines de ses limites, voire de ses contradictions. Donc pour mettre à l'épreuve sa validité : n'est-il pas aujourd'hui si fréquemment et si mollement convoqué qu'il perd sa spécificité ? Qui plus est, n'est-il pas débordé, voire dépassé par d'autres conceptions et surtout, par d'autres pratiques sociales, d'autres manières de vivre?

A cette fin, contre certains réflexes de pensée, nous avons pris l'initiative, qui pourra heurter les méthodologues orthodoxes, d'enquêter au-delà de nos champs usuels. D'ouvrir la fenêtre, de regarder et d'écouter ce qui se fait et se dit ailleurs, dans d'autres disciplines et secteurs d'activités.

\footnotetext{
${ }^{5} \mathrm{http}: / /$ contextualisations.sciencesconf.org/resource/page $\mathrm{id}=6$
} 
En précisant bien que notre intention sera toujours de contribuer à la didactique du plurilinguisme, et plus exactement du plurilinguisme issu de la migration, nous avons pris le parti d'interroger l'actualité de deux disciplines a priori étrangères à notre champ, la littérature d'aujourd'hui et l'histoire de l'art contemporain, afin de voir comment ces deux champs, particulièrement attentifs au présent, pensent différemment, jusqu'à les infléchir, les postulats les plus établis de la postmodernité régnante. Comment la littérature (écrivains et critiques) et comment les artistes impliqués dans les flux et les échanges mondiaux modélisent-ils, exemplifient-ils ce qui est au cœur des réflexions sur les contextes didactiques, à savoir les formes et les manières de vivre, d'endosser l'individuation et l'appartenance ou de concevoir l'altérité, la différence?

« Parce qu'elle n'accepte pas la délimitation bien définie et éprouvée de l'objet, l'entreprise interdisciplinaire doit tout d'abord avoir pour effet de décevoir » (Blumenberg, $2005: 8$ ). En assumant le risque de surprendre et de troubler les habitudes de pensée, en conviant donc le lecteur à sortir un instant de sa zone de confort habituel, en espérant que ce détour par d'autres disciplines permettra de revisiter certains de nos postulats implicites, nous visons à nuancer la manière de décrire notre environnement et, donc, d'agir sur lui.

Dans les faits, après un inventaire des déclinaisons actuelles de l'identité que nous établirons à partir de Rosie Carpe, un roman de Marie Ndiaye (2001), et d'un essai de Pierre Bayard (2010), Et si les ouvres changeaient d'auteur?, nous recourrons à la description des enjeux et des formes de l'art contemporain international qu'a récemment proposé l'historien d'art Nicolas Bourriaud (2010), pour confirmer aussi bien la prégnance de la postmodernité que certaines de ses insuffisances à penser notre présent, puis nous appliquerons ces définitions de notre épistémologie que nous estimons plus ajustées à la réalité actuelle à une démarche d'enseignement plurilingue qui a été récemment proposée en Suisse, à l'intention des élèves de l'école primaire. De La condition postmoderne convoqué au début de cette étude à un Imagier plurilingue destiné à des élèves entrant dans la lecture et l'écriture, c'est peu dire que le lecteur sera amené à des changements de perspective considérables et nous sollicitons autant sa bienveillance que sa curiosité.

\section{Un laboratoire des postures identitaires les plus actuelles}

La littérature sera notre premier repère, en tant qu'elle modélise des formes d'individualisation et d'appartenances actuelles. Dans Rosie Carpe, de Marie Ndiaye, les personnages principaux voyagent entre Antony en France, et Pointe-à-Pitre en Guadeloupe.

Au-delà de l'intérêt que constitue en soi l'exploration de plusieurs lieux, la transition géographique, le voyage donnent l'occasion d'expérimenter les fluctuations de l'identité. Deux personnages retiendront notre attention tout particulièrement : Danielle, la mère de Rosie, ainsi que Rosie Carpe, l'héroïne éponyme du roman.

En émigrant en Guadeloupe afin de couler une retraite dorée, Danielle échange son prénom contre celui de Diane. Au grand étonnement de sa fille lorsqu'elles se retrouvent, l'identité nominale ne semble jouer qu'un rôle décoratif pour l'aïeule, qui a choisi un nouveau nom pour des raisons esthétiques. Plus étonnante encore, et plus intéressante pour notre problématique, une seconde transformation, physique celle-là, se superpose au renouvellement de l'identité nominale : (Danielle-) Diane rajeunit, sans que cela soit dû à une intervention externe, de type chirurgical ou médical. Ce processus semble provenir mystérieusement de l'intérieur. Mais 
alors que Diane redécouvre une vie pleine de promesses, quelque chose en elle effraie : «En réalité, elle n'a ni vingt ans ni trente mais bien plutôt cinquante. Mais elle est jeune. Tout en elle, à quelques détails près, est monstrueusement jeune » (Ndiaye, 2001 : 190).

L'autre personnage archétypal est Rosie Carpe, qui incarne le doute et, paradoxalement, la stabilité identitaire. A plusieurs occasions dans le roman, Rosie témoigne d'une ignorance préoccupante de sa propre identité : « Rosie n'était même pas certaine d'être bien la fille qu'on appelait Rosie » (Ndiaye, $2001: 59$ ) ou plus loin, lorsqu'elle lit son prénom officiel sur une liste de résultats d'examens : " Mais qui est Rose-Marie ?» (Ndiaye, 2001 : 61). Cependant, ces périodes d'incertitude alternent avec d'autres qu'on pourrait qualifier de solidité identitaire : "Elle savait qu'elle était Rosie Carpe et que c'était bien elle, à la fois Rosie et Rosie Carpe, qui marchait en ce moment d'un pas tranquille [...]. Et elle était maintenant Rosie Carpe sans doute possible » (Ndiaye, 2001 : 127-128) ou encore : « Rosie tourna la tête pour se voir dans la vitrine. Et elle vit une grande silhouette rouge aux épaules droites, au ventre tendu, au visage hâlé tout éclairé de jubilation triomphante. Et elle se dit sans hésitation que c'était là Rosie Carpe, c'est-à-dire assurément, elle-même » (Ndiaye, 2001 : 283).

On découvre deux postures distinctes. La première tente de se débarrasser d'une «identitéfardeau »: alors que Diane s'installe dans sa nouvelle identité, elle sacrifie les liens qui la rattachaient à sa vie antérieure, en éradiquant de manière volontaire et définitive certains souvenirs constitutifs, filiaux notamment. La deuxième se raccroche à une «identité-racine ». Malgré les épisodes de doute, malgré ceux où Rosie aimerait imiter sa mère et couper le lien avec son propre fils, son identité et son passé la rattrapent, inoubliables.

Analogiquement à Rosie Carpe, d'autres œuvres littéraires de l'extrême contemporain mettent en scène deux modèles de l'identité que commente parmi d'autres le sociologue Jean-Claude Kaufmann. Son hypothèse : les processus psychologiques de constitution de l'individu ne peuvent être dissociés des structures sociales dans lesquelles ils prennent vie. Au risque de paraître schématique, voilà une opposition qui éclaire le statut des personnages romanesques. Dans la première moitié du $\mathrm{XX}^{\mathrm{e}}$ siècle, les individus appartenaient à des « ensembles sociaux relativement stables ", institutionnalisés et orientés par une force étatique (ou morale, ou religieuse) (Kaufmann, 2004: 27). Au tournant des années 1960, les communautés se désagrègent (et l'on retrouve là, sous une autre terminologie, sous une autre compréhension, la faillite des méta-récits lyotardiens) : l'individu est alors contraint de s'auto-définir et il ne peut plus s'appuyer sur une instance extérieure à lui (Kaufmann, $2004: 58$ ).

Ces quelques indications relatives à l'identité en contexte postmoderne permettent de lire avec plus de nuances les métamorphoses ou ancrages identitaires romanesques. Diane se fond dans une sorte d'indifférenciation pathologique, en défiant la logique linéaire de la temporalité et du vieillissement. Incarnerait-elle le miroir grossissant d'une réalité sociale, l'ouverture proclamée à tous les possibles ? Rosie Carpe, quant à elle, oppose une résistance à ce tropisme postmoderne, travaillant à rétablir (en vain) unité et assise dans son entourage. Selon Kaufmann, la construction identitaire ne serait plus à chercher dans une stabilité qui ne saurait être que fantasme aujourd'hui, mais bien plutôt dans une configuration en îlots de sens, cohérents mais mutants, qui contribueraient à forger un individu multiple et fragmenté, prêt à endosser le rôle le plus adéquat requis par la situation. Prêt aussi, à en changer. Ainsi, la compulsion à revêtir des identités multiples n'est plus seulement le symptôme d'un trouble privé, mais vaut pour la société contemporaine tout entière, elle-même frappée par la métamorphose et la discontinuité, pour ne reprendre que ces traits de la postmodernité telle que définie plus haut. 
C'est également sur une forme de discontinuité que réfléchit le critique Pierre Bayard, dans un récent essai, où il récrit des épisodes célèbres de l'histoire littéraire en attribuant des œuvres phares à des auteurs inédits. Deux des catégories qu'il explore s'appuient sur des faits avérés (Romain Gary qui a écrit sous sa propre identité et sous celle de Emile Ajar, Boris Vian qui s'est dissimulé sous le pseudonyme de Vernon Sullivan) ou sur des controverses, plus ou moins célèbres, qui ont interrogé la réelle généalogie de certaines œuvres et de leur auteur présumé erroné (Homère, Shakespeare, Molière).

Mais c'est bien la troisième catégorie qui nourrit notre questionnement autour du plurilinguisme et de ses contextualisations didactiques. Dans la dernière section de son ouvrage, Pierre Bayard propose au lecteur un changement bien plus radical, « en brisant définitivement le tabou qui voudrait qu'une œuvre ait un seul créateur » (Bayard, 2010 : 14) : autrement dit, en attribuant à des romans dont l'auteur n'est sujet à aucune indétermination, un autre auteur. On perçoit une analogie avec l' «erreur formatrice», qui permet une nouvelle découverte, un changement de paradigme - bien que l'erreur soit ici volontaire et assumée : " en modifiant l'auteur en partie ou en totalité, nous nous mettons dans les dispositions psychologiques de percevoir l'œuvre comme des lecteurs d'autres temps ou d'autres pays pourraient le faire, et l'erreur assumée est ici un autre nom pour l'ouverture d'esprit» (Bayard, 2010 : 12). «Pourquoi une œuvre n'aurait-elle qu'un seul auteur?" se demande Bayard. La substitution d'identités d'auteurs mettrait au jour des significations inattendues contenues dans les œuvres, mais que leur filiation classique ne permet pas de tisser.

Un exemple concret: Bayard propose un pastiche d'une anthologie de littérature où l'on découvre L'Etranger de Franz Kafka : « On a longtemps mis à part L'Etranger dans l'œuvre de Kafka, comme s'il s'agissait d'un roman trop atypique pour disposer du même statut que les autres et comme s'il appelait de ce fait un traitement autonome. La gêne qu'il suscite régulièrement chez les critiques est telle qu'il n'est pas rare que leurs travaux sur Kafka ne le mentionnent même pas » (Bayard, 2010 : 83). Ainsi, Meursault, le héros de Camus, est placé en droite ligne avec Joseph K., le héros du Procès de Kafka : pour le critique littéraire, le geste criminel de Meursault est kafkaïen et la parodie de justice qu'on lit dans L'Etranger est un indice supplémentaire qui justifie cette filiation.

Changer d'auteur permet de poser un nouveau regard sur l'œuvre littéraire : la question de la justice se trouve certes présente dans le roman de Camus ; mais considérer L'Etranger comme le prolongement de l'œuvre romanesque de Kafka infléchit l'interprétation avec promesses, soumet à notre sagacité d'heureuses conjonctions de sens inattendues. Car il s'agit plus de superpositions d'auteurs, de colorations qui se mêlent aux précédentes, de sens qui se révèlent en se conjuguant, que d'une disparition de Camus au profit du seul Kafka. La substitution d'auteurs requiert de revisiter la manière dont les œuvres sont reçues, c'est-à-dire, dans une vision relativiste, d'étudier comment se construit à chaque fois, en fonction de l'identité prêtée à l'auteur, un univers de réception différent.

Poursuivant notre succincte typologie des identités d'aujourd'hui, à l'identité-fardeau et l'identité-racine, on ajoutera ici deux figures. L' « identité-costume », tout d'abord, qui permet de substituer un auteur à un autre, mais pas de manière anarchique ou aléatoire : en respectant certaines règles de cohérence thématique ou d'époque. Kafka pour Camus, oui, mais pas Marie Ndiaye. L' « identité-prison », ensuite, repoussoir contre lequel lutte Pierre Bayard, lorsque la stabilité est gage d'enfermement et de paralysie, non de fondation à partir de laquelle on construit. 


\section{Une postmodernité généreuse mais embarrassée}

Des réflexes de repli sur l'identité unitaire à ses éclatements, angoissants peut-être mais aussi libératoires, en facettes multiples, les modélisations de l'identité par l'écriture et la critique littéraires résument les mutations épistémologiques et sociales qui nous ont fait glisser de la modernité à la postmodernité et ils nous permettent de préciser quelques caractéristiques de ce paradigme, qui nous pense et pense des institutions comme l'École.

Ne perdant pas de vue notre objectif, qui est bien d'améliorer la compréhension des contextes didactiques : (i) en ajustant notre description des rapports d'appartenance d'un individu à un ou à plusieurs milieux et cultures, et (ii) en examinant les échanges entre un contexte donné, local, et les autres, nous compléterons brièvement la définition de la postmodernité afin de tester sa capacité à décrire notre présent.

Pour décrire la postmodernité par rapport à l'ère moderne qui l'a précédée, on peut convoquer les vertus de métaphores aussi connues que pédagogiques : quand la modernité se représente commodément par l'image de l'arbre, la postmodernité, depuis Deleuze et Guattari (1980), se commente souvent par celle du rhizome.

L'arbre, de ses racines à son fût, ses branches et ses fruits, métaphorise bien et la société et l'individu en régime moderne. La modernité répond à un Projet de société, garanti, dirigé, vectorisé par des valeurs majuscules, haut dressées, à visées universalistes, messianiques, conquérantes donc (la Religion, l'Histoire, le Progrès, etc.) : s'il fallait un exemple, pensons à l'œuvre de Paul Claudel, qui arpente et inventorie la réalité chatoyante du monde en intendant de Dieu. En régime moderne, le Temps est la dimension privilégiée : le Passé étant dépositaire d'une vérité qui s'accomplira dans le futur, les activités humaines sont finalisées. Puisque le Passé est à la fois prescripteur et promesse (de progrès, de salut, etc.), la transmission des valeurs d'une génération à l'autre est essentielle et plutôt aisée. Une échelle explicite de ces valeurs organise et règle la place et les activités des individus, lesquels disposent d'une autonomie restreinte. Le primat du corps social et l'organisation holiste ancrent l'individu dans ses déterminismes (être né là, à ce moment) tout en lui assignant une perception identitaire plutôt stable et unique de sa propre identité.

Par rapport à l'arbre, le rhizome s'élève peu, car c'est une tige horizontale souterraine, qui croît à plat, par nœuds et bourgeons : aussi cette image a-t-elle semblé adéquate pour qualifier nos sociétés postmodernes, relativistes, sans projet unitaire, sans finalités consensuelles. Quand la modernité privilégiait la verticalité et le temps, la postmodernité, vouée au pointillisme du présent, procède de l'Espace, qui régit des rapports contigus, simultanés ou juxtaposés. Le passé dépositaire de valeurs ne servant plus de référence, la transmission est devenue difficile, sinon problématique. Le rapport aux connaissances et savoirs s'en voit modifié et il change d'autant plus vite que se densifient et se globalisent les accès aux informations et aux ressources. Le rhizome offre aussi ses analogies pour décrire l'individu postmoderne. Alors que les racines de l'arbre sont vectorisées, qu'elles convergent, qu'elles sont téléologiques, celles $\mathrm{du}$ rhizome ne tendent pas à un projet unique. L'organisation sociale postmoderne est faiblement collective, elle est plutôt un agrégat d'individus, modérément reliés. Chacun de ceux-ci gagne en initiative et liberté ce qu'il perd en sécurité et stabilité : comme la littérature nous l'a rappelé, l'identité aujourd'hui est autant vécue et perçue sur le mode du manque et de la perte que de l'autonomie ou du gain par adjonctions, ajouts, voire métamorphoses. 
Si la postmodernité décrit semble-t-il assez précisément les sociétés actuelles et notamment ce que de celles-ci l'École et l'Éducation cristallisent, le paradigme doit être cependant discuté dans ses présupposés aussi bien que dans sa capacité à commenter les manières les plus contemporaines qu'a un individu de vivre son rapport aux contextes et à autrui. Sans remonter aux critiques qui ont accompagné sa définition et son histoire (Habermas, 1981, qu'on a évoqué plus haut, mais aussi Meschonnic, 2009 ; Dufour, 2011), la postmodernité a peu interrogé son ethnocentrisme (européen, «occidental») et elle ne s'est pas suffisamment prémunie contre le réflexe historique du continent de généraliser et d'universaliser. Le relativisme qui est à la base de la postmodernité est donc contredit par les traditions modélisantes et universalisantes des cultures européennes. De ce point de vue, la postmodernité serait encore un avatar des universalismes historiques anciens. Par exemple, quand Edgar Morin professe que «l'idée de France est une idée qui comporte désormais, dans sa singularité même, l'idée d'universalité » (Morin, 2008: 166), c'est encore et toujours la vieille idéologie littéraire et messianique française qui agit, celle-là même qui a instauré, jadis, la République mondiale des Lettres (Casanova, 1999). Il s'ensuit que le discours de la postmodernité n'a sans doute pas vraiment intégré cela même qu'il décrit, à savoir la fin des ancrages identitaires (métaphorisons encore : la fin de la racine unique). A tout le moins, les tensions restent permanentes entre le relativisme (de telle culture, de telle appartenance) et le réflexe d'ériger une culture particulière en parangon. Ainsi, la postmodernité telle qu'elle semble comprise et pratiquée ne serait pas, au sens propre du mot, relativiste, elle ne mettrait pas vraiment en relation équilibrée les cultures. Dans les faits, elle ne résout pas l'alternative entre le repli identitaire, peureux et défensif, et une compréhension sereine du multiculturalisme et des identités panachées. Par exemple, la Suisse, qui se félicite volontiers de son fédéralisme multiculturel et plurilingue, sécrète un nationalisme et une xénophobie qui recueillent l'approbation de près de $30 \%$ de ses citoyens. Une autre critique adressée à la postmodernité implique encore son relativisme, mais cette fois dans un autre sens du mot : sa mollesse, c'est-à-dire sa peine à établir que tout ne se vaut pas, aurait fait le lit du néolibéralisme mondial, lequel s'est imposé d'autant plus facilement que la dérégulation morale (diminution du pouvoir régulateur de l'Etat, précarisation de l'emploi, etc.) qu'il instaurait s'est faite en flattant l'hédonisme consumériste des individus (Dufour, 2007).

Résumons cette synthèse trop condensée de la postmodernité - nous en assumons donc la simplification caricaturale - par le rappel d'une situation d'enseignement aujourd'hui banale : alors que le relativisme postmoderne repose sur des postulats a priori généreux et compréhensifs de la diversité, de l'altérité, de la différence, aucune des communautés francophones n'a de proposition vraiment convaincante pour articuler et penser de manière harmonieuse et non discriminante l'intégration - ou selon le nouveau mot estimé politiquement plus correct en Suisse, l'inclusion - scolaire et sociale des élèves issus de la migration. Si nos structures d'accueil s'appliquent assez positivement aux élèves issus des cultures et langues nationales ou des pays aux économies et cultures estimées similaires, elles restent peu efficaces, peu altruistes, pour accueillir les migrants économiques ou politiques (Europe de l'Est, Afrique, Asie, etc.). Les principaux modèles actuellement disponibles, celui, britannique, de la juxtaposition des cultures ou celui, français, de l'intégration, ont montré leurs défauts et leurs limites et la Suisse, malgré ses efforts, malgré sa richesse, malgré des démarches d'enseignement parfois originales et quel que soit son système scolaire (l'Éducation helvétique est dans une large mesure une prérogative de chaque canton), continue à discriminer les élèves de la migration, qui n'ont de loin pas les mêmes chances de formation que les autres. Si tout ne s'explique évidemment pas par la manière de penser les cultures, il n'en reste pas moins que le système qui, sur le papier et en réalité accueille (Plans d'études, classes d'accueil, cours de langues intensifs, démarches pour valoriser les langues et cultures d'origine, etc.), continue de 
hiérarchiser les cultures et peine, malgré des efforts louables, à sortir les représentants de ces communautés d'une image négative d'elles-mêmes.

Quand on lit la situation de cet élève péjoré avec une lunette postmoderne, on constate que le climat ambiant lui a fait miroiter, en théorie, une hybridation, une cohabitation harmonieuse de ses différentes cultures, alors qu'en réalité, elles sont hiérarchisées, les plus puissantes d'entre elles (du point de vue historique, géostratégique, économique) revendiquant un accès privilégié à l'universel. Ceci est cependant moins discriminant que sa traduction sur le plan identitaire, sur la manière que cet élève a de vivre son rapport à lui et à autrui. A ce niveau, la postmodernité émet un message ambivalent et qui est l'une des sources possibles des nombreux malaises identitaires actuels : même quand elle invite et encourage l'hybridation culturelle, elle postule, contre les risques et l'anonymat de la culture mondialisée, la nécessité et les vertus de l'enracinement identitaire, de la différence. Oserions-nous une nouvelle image ? Nous parlerons en ce cas du label A.O.C. (Appellation d'Origine Contrôlée) : chacun doit choyer sa racine, cultiver sa singularité. Autrement dit, la postmodernité ne paraît pas encore vraiment émancipée de l'ontologie de la racine héritée de la modernité et son relativisme est loin d'être achevé.

\section{De la postmodernité à l'altermodernité}

Dans un récent essai intitulé Radicant, Pour une esthétique de la globalisation, Bourriaud (2009), critique d'art, conservateur et philosophe, a proposé un cadre théorique à ses précédents travaux qui étaient jusqu'alors plutôt des descriptions organisées des activités artistiques internationales les plus actuelles. Vous l'avez bien lu : oui, cet ouvrage s'intéresse de près aux formes d'art contemporaines, aux accrochages, aux installations, aux performances, qui se tiennent dans des lieux institutionnalisés (musées d'art contemporain, galeries, etc.) ou de circonstance (parcs publics, centres commerciaux, aéroports, etc.) : exactement à tout ce que la majorité d'entre nous ne veut ou ne sait voir, lire, comprendre ou situer. Aux artistes, peintres, vidéastes, performers qui, généralement, égarent ou déçoivent le sentiment du visiteur lambda, le plus souvent encore moulé par l'esthétique classique et par le primat du beau.

En acceptant ici le défaut anachronique qui tendra à considérer les artistes actuels encore comme ces pionniers, ces visionnaires ou prophètes que le romantisme et l'art moderne ont jadis mythifiés (artistes maudits, impressionnistes, fauvistes, cubistes, etc.), à savoir à les approcher comme des précurseurs des futures manières de penser et d'agir, nous allons suivre certains mouvements de cet art contemporain international que Bourriaud tient pour « un laboratoire des identités » $(2009: 58)$.

Voici donc Mike Kelley qui, avec son installation Frame and Fame, peut, par ricochet, discuter ses origines irlandaises en reconstituant... un monument chinois situé près de son actuel ancrage, à Los Angeles (d'après Bourriaud, 2009 : 59 et 84).

Puis voici Road to exile (2008) de Barthélemy Toguo, qui représente une barque chargée de ballots colorés et de plastiques, symbolisant, on l'a compris, la périlleuse réalité des boat people africains traversant la Méditerranée.

Ou voici, dans un sens proche, mais pas toujours autant explicitement politisé, les différentes performances, les nombreux accrochages, les multiples voyages qu'accomplit la coréenne Kim Sooja avec des costumes ou étoffes traditionnels de son pays d'origine (Bottari, 2000 ; Bottari, 2009). 
Dans la typologie de Bourriaud, ces quelques artistes-ci, emblèmes des millions d'immigrés ou d'exilés que compte la planète, mais aussi des nomades ou des simples touristes, sont des voyageurs, des exotes, et leurs performances sont majoritairement des trajets entre différents lieux, cultures, espaces. Leurs activités, leurs œuvres, leurs intentions semblent se conformer en tous points à la doxa postmoderne qui postule l'hybridation à la différence capitale près qu'ils se sont libérés de l'ontologie de la racine et que, en général, ils se sont émancipés des instances de consécration artistique que représentait « l’Occident» et sa prétention universaliste.

«Ainsi les artistes d'aujourd'hui expriment moins la tradition dont ils sont issus que le parcours qu'ils accomplissent entre celle-ci et les divers contextes qu'ils traversent, en réalisant des actes de traduction. [...] Il [l'artiste] ne recherche pas un état idéal du Moi, de l'art ou de la société, mais organise les signes afin de multiplier une identité par une autre. [...] On peut emporter avec soi des fragments d'identité, à condition de les transplanter sur d'autres sols et d'accepter leur permanente métamorphose - une sorte de métempsychose volontaire, préférant à toute incarnation le jeu des panoplies successives et des abris précaires » (Ibid. : 5859).

Dans le sens des images qu'il a commencé à filer, Bourriaud propose une nouvelle métaphore pour illustrer un nouveau paradigme. Après l'arbre de la modernité, puis le rhizome postmoderne, voici donc le radicant de l'altermodernité.

«L'immigré, l'exilé, le touriste, l'errant urbain sont [...] les figures dominantes de la culture contemporaine. L'individu de ce début de XXI siècle évoque, pour rester dans un lexique végétal, ces plantes qui ne s'en remettent pas à une racine unique pour croître mais progressent en tous sens sur les surfaces qui s'offrent à elles en y accrochant de multiples pitons, tel le lierre. Celui-ci appartient à la famille botanique des radicants, qui font pousser leurs racines au fur et à mesure de leur avancée, contrairement aux radicaux dont l'évolution est déterminée par l'ancrage dans un sol. La tige du chiendent est radicante, tout comme les drageons du fraisier. [...] Il [le radicant] se traduit dans les termes de l'espace où il évolue. Par sa signification à la fois dynamique et dialogique, l'adjectif radicant qualifie ce sujet contemporain tenaillé entre la nécessité d'un lien à son environnement et les forces $\mathrm{du}$ déracinement, entre la globalisation et la singularité, entre l'identité et l'apprentissage de l'Autre. Il définit le sujet comme un objet de négociations » (Ibid. : 58).

Ce paradigme original de l'altermodernité, qui prend acte de la globalisation et de l' « interconnectivité » généralisée, doit affronter l'indifférenciation, l'anonymat et l'entropie qui en résultent et qui abîment toute individuation, tout particularisme. A cette fin, et toujours en observant des démarches artistiques contemporaines, Bourriaud exhume le concept du Divers que l'écrivain-voyageur Victor Segalen (1878-1919) avait conçu au moment de ce qui était alors une première forme de globalisation (l'internationalisme, le cosmopolitisme), au moment de la fin présumée de l'exotisme et, partant, d'une homogénéisation du monde (Cordonier, 1996). "L'esthétique du Divers » qu'a conçue et pratiquée Segalen est convoquée par Bourriaud comme une méthodologie pour apprécier les très fines différences culturelles qui restent possibles malgré la globalisation, de les faire jouer en montrant « la coprésence des points de vue au sein d'un espace multifocal » (Ibid. : 76). On le devine, l'altermodernité que mettent en pratique les artistes contemporains repose obligatoirement sur la prise en compte de la réalité géoéconomique et culturelle du monde actuel. Dans le sillage d'Edouard Glissant, 
puis de Bernabé, Chamoiseau et Confiant, Bourriaud convoque et commente la nécessaire « créolisation, à la fois comme phénomène historique, formule de mélange et mode de pensée. [...] Contre la standardisation obligatoire, la créolisation ramifie à l'infini les discours culturels et les brasse dans un creuset minoritaire, les restituant, parfois méconnaissables, sous la forme d'artefacts désormais coupés de leurs origines » (Ibid. : 83-84).

Contre la vieille sujétion à l'universalisme occidental, contre les ambiguïtés de la postmodernité qui a imposé cette mauvaise alternative entre l'enracinement identitaire et l'anonyme uniformisation engendrée par la globalisation économique, de nombreux artistes contemporains, mais aussi des écrivains et des critiques (Marie Ndiaye, Pierre Bayard), montrent ou modélisent donc comment « mettre en scène, mettre en route ses racines dans des contextes et des formats hétérogènes " (Idem : 23) et ils annonceraient ainsi une nouvelle manière de lire et de vivre notre temps, l'altermodernité :

\begin{tabular}{|c|c|c|}
\hline \multicolumn{3}{|c|}{ Paradigmes épistémologiques } \\
\hline 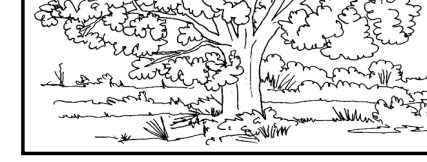 & & $=$ \\
\hline http://www.jecolorie.com/ & http://ceurvorst.com/ & http://www.paysage-patrimoine.eu/ \\
\hline Arbre & Rhizome & Radicant \\
\hline MODERNITE & POSTMODERNITE & ALTERMODERNITE \\
\hline $\begin{array}{l}\text { Verticalité } \\
\text { Temps } \\
\text { Transmission } \\
\text { Hiérarchie des valeurs } \\
\text { Projet, Finalité } \\
\text { Universalisme } \\
\end{array}$ & $\begin{array}{l}\text { Horizontalité } \\
\text { Espace } \\
\text { Communication } \\
\text { Juxtaposition } \\
\text { Réseau, Simultané } \\
\text { Relativisme }\end{array}$ & $\begin{array}{l}\text { Postmodernité globalisée sans } \\
\text { l'ontologie de la racine : } \\
\text { - intersubjectivité } \\
\text { - réacclimatation } \\
\text { - traduction } \\
\text { - trajets, voyage }\end{array}$ \\
\hline
\end{tabular}

Tableau 1 : Paradigmes épistémologiques

\section{Une démarche didactique altermoderne : L'imagier plurilingue}

Après cette excursion en dehors de nos disciplines usuelles et de nos habitudes de pensée, il est temps de retrouver notre préau, de revenir à nos préoccupations didactiques lesquelles, rappelons-le, concernent la diversité culturelle des contextes plurilingues et, concrètement, visent à valoriser les langues d'origine des élèves, plus particulièrement des élèves issus de la migration. Après les écrivains, les philosophes et les artistes, place à ces extrêmes contemporains que sont les jeunes élèves.

La démarche didactique que nous allons décrire a des qualités intrinsèques qui mériteraient une minutieuse présentation: on ne l'entreprendra toutefois pas ici parce que, rappelons-le, notre objectif principal est d'inviter les enseignants, les didacticiens, les sociolinguistes,... à modifier leur lecture, leur manière d'observer et de commenter les élèves allophones dans des situations d'enseignement/apprentissage du français ou d'une autre langue de scolarisation. À condition que la démarche que nous résumerons entretienne des rapports d'analogie avec celles que nous avons décrites en passant par la littérature et les arts contemporains, cet unique exemple nous fournira tout ce qui est nécessaire à notre objectif. 
«Qu'est-ce qui est semblable dans notre classe et dans toutes les autres classes ? » : en partant de cette question, deux chercheures en didactique du plurilinguisme, aidées de trois enseignantes, ont proposé à des élèves de trois classes enfantines (cycle 1) du canton de Genève de construire un imagier plurilingue. Dans cet imagier sont représentés le français, langue officielle de l'école, ainsi qu'un choix de dix-sept langues parlées par les élèves des classes en question (par ordre alphabétique : l'albanais, l'allemand, l'anglais, l'arabe moderne, l'espagnol, l'italien, le kurde kurmandji, le kurde sorani, le mooré, le polonais, le portugais, le serbe, le somali, le tagalog, le thaï, le turc). Une forte volonté d'impliquer les élèves animait le projet : en convoquant leur propre expérience de l'école et de la salle de classe, ainsi qu'en travaillant par généralisation, les adultes ont demandé aux très jeunes élèves : que trouve-t-on dans notre classe ? Et dans toutes les classes ? Par cooptation, les enfants ont sélectionné douze mots qui désignent des objets appartenant à la vie de la classe : le tableau, le pupitre, la chaise, la règle, les ciseaux, le livre, le bâton de colle, la feuille de papier, le crayon, le stylo feutre, le pinceau, la gomme. Ces mots, agrémentés d'un chiffre (un tableau, deux pupitres, trois chaises, etc.) ont ensuite été illustrés par les enfants eux-mêmes, ce qui rapproche cette démarche de celles qui recourent, avec de jeunes enfants de classes multiculturelles, au dessin « comme élément d'une herméneutique du sujet plurilingue » (Molinié, 2009 ; Moore et Castellotti, 2011). A succédé à ces étapes, la phase de traduction des mots du français en chacune des dix-sept langues : c'est ici qu'on a requis l'aide des parents allophones ou bilingues, ainsi que celle de bénévoles qui ont enregistré les mots dans les langues originales. Enfin, les chercheures ont rédigé les textes du livret pédagogique et elles ont mis en ligne un site internet contenant des activités à tester en classe, à la maison, en bibliothèque.

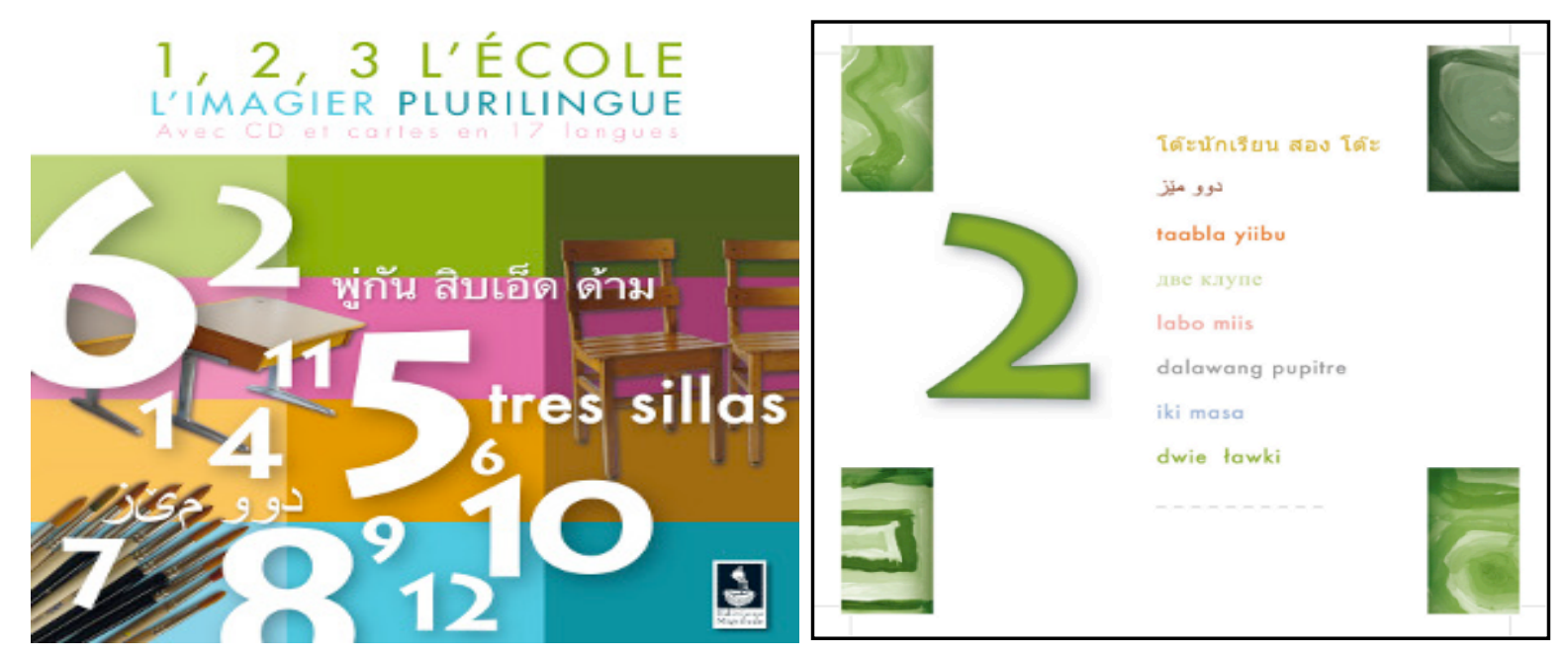

Tableau 2 : L’imagier plurilingue (couverture et carte « 2 pupitres »)

Les chercheures et les enseignantes ont bien sûr dû procéder à des choix :

- Quel système d'écriture privilégier pour une langue telle que le kurde - qui en comporte deux, le sorani et le kurmnadji ?

- Quel arabe retenir? L'arabe classique ? L'arabe moderne? L'arabe qui est parlé dans les pays du Maghreb?

- Quels niveaux de langue accepter dans les traductions?

- Comment intégrer les usages régionaux, les dérivés locaux ?

- Que faire avec les langues, telles que le mooré, qui n'ont pas été encore stabilisées à l'écrit? 
- Comment contourner la difficulté de travailler avec des langues qui ne comportent par de traduction pour tous les mots retenus ? Par exemple, "bâton de colle » manque d'équivalent dans certaines langues.

Cette liste de questions n'est évidemment pas exhaustive ; elle possède toutefois l'avantage de «typologiser» quelques difficultés auxquelles se sont heurtés les élèves, les parents, les enseignantes et les chercheures. De rappeler également que traduire revient à faire des choix, à trancher. Et à assumer, à défendre les options retenues.

Arrêtons-nous un instant sur la phase de traduction de l'imagier, qui constitue un exemple modélisant du radicant de Nicolas Bourriaud. Le principe était le suivant : chaque élève rentrait chez lui avec un mot en français à traduire dans sa langue première avec l'aide de ses parents, puis il ramenait le mot ainsi traduit à l'école. Autrement dit, on retrouve dans ce projet autour du plurilinguisme la notion de trajet : ramener la culture du pays d'accueil dans son milieu originel, familial ; et stimuler, de ce fait, une réflexion linguistique et culturelle ; puis, en sens, contraire, en direction de l'école, emporter des bouts de sa culture et les déposer dans un contexte culturel différent, celui de l'école et de sa norme.

Parallèles : à l'artiste globalisé qui transporte des fragments tangibles de sa culture première dans des contextes autres pour les faire jouer, pour les frotter à d'autres réalités et cultures correspond l'enfant qui transporte entre son domicile et l'école des mots (écrits ou par oral) pour les mettre en correspondance ou tensions avec leurs équivalents dans d'autres langues. Ce trajet, géographique, matériel, linguistique mais également symbolique peut être lu comme un minuscule équivalent des trajets artistiques qui illustrent, aujourd'hui, une manière, comme le dit Bourriaud, de mettre en route ses racines et d'instaurer un dialogue décomplexé avec d'autres racines, d'autres altérités. Matériel, linguistique, mais également symbolique, ce trajet effectué par les élèves peut être lu comme l'un des traits caractéristiques de l'altermodernité. Toujours par analogie avec le monde de l'art contemporain, on dirait même que le trajet en soi, ou le processus d'élaboration, est aussi important que le résultat, dans la mesure où il participe à niveau égal à la construction du sens.

\section{Conclusion}

Est-ce là tout? Tout ce long excursus pour arriver à lire un peu différemment une sensibilisation au plurilinguisme ? Outre qu'il est méthodologiquement peu orthodoxe, notre détour aboutit à un parallèle qui semble incongru entre des artistes contemporains qui mettent en scène des trajets et des transports d'objets et des jeunes élèves qui font des allers et retours entre leur domicile et l'école, lestés de quelques banals mots à traduire. Par ailleurs, la manière de commenter ces voyages change apparemment peu si on les lit, soit à la manière usuelle postmoderne, soit du point de vue nouveau de l'altermodernité. Dans les deux cas, l'identité personnelle est vécue sur le mode de la pluralité, de la diversité, du panachage.

N'y aurait-il cependant qu'un simple changement de modalité dans la manière de dire ces expériences que la manière de les vivre pourrait s'en trouver changée. Pour la postmodernité, qui est encore notre cadre de pensée le plus familier, la fascination pour l'origine, pour la racine est telle que la norme d'un parcours identitaire consiste encore et toujours à re-trouver son identité. S'il n'est pas question de nier ici les douleurs et désarrois des exils politiques ou économiques de millions d'êtres humains, le standard de l'identité postmoderne est presque toujours caractérisé par la perte ou le manque parce que, dès son préfixe, la postmodernité reste tributaire de cela même à quoi elle prétend succéder, une modernité dont l'universalisme 
cautionnait une mythification, voire un fétichisme de la racine. Il s'ensuit qu'en régime postmoderne, les bonnes pratiques et conduites pédagogiques sont toujours peu ou prou réparatrices, consolantes : il convient d'aider un élève, un individu à vivre harmonieusement les différentes facettes ou composantes identitaires et à tenter de réduire les hiérarchies entre elles. Or cette aporie entre le dialogue interculturel et l'essentialisme de la racine neutralise les gestes didactiques les mieux intentionnés.

Si l'on ose pousser à bout notre pensée, on pourrait même soupçonner que nous, les enseignants et didacticiens travaillant sur l'inclusion, nous sommes peu ou prou les otages d'une conception encore majoritairement doloriste de l'identité et que nous pourrions de ce fait plus freiner que stimuler les jeunes à se dégager vraiment de l'essentialisme de la racine et à vivre un rapport à l'appartenance autre, décomplexé, à la manière de ce que modélisent des écrivains ou de ce que pratiquent des artistes contemporains.

Emblématisée par ces créateurs qui mettent en tension de manière critique mais non nécessairement affligée et abîmée des fragments culturels dans une globalisation qu'ils dénoncent mais qui ne les inhibent pas, l'altermodernité, elle, prend simplement acte de la mobilité généralisée des personnes, des biens, des savoirs, des informations ou des services et pense l'identité comme un geste de traduction généralisée et comme des actes de réenracinements successifs. La modalité, en ce cas, est tout autre et les trajets et les traductions, comme l'exemplifie à sa manière L'imagier plurilingue, peuvent commencer à être vécus comme des accroissements identitaires, et non plus éprouvés sur le mode de la réparation.

\section{Références bibliographiques}

Bauman, Z. (2006 [2005]). La vie liquide. Rodez : Le Rouergue / Chambon.

Bayard, P. (2010). Et si les æuvres changeaient d'auteur? Paris : Minuit.

Bernabé, J., Chamoiseau, P. et Confiant, R. (1989). éloge de la créolité. Paris : Gallimard.

Blanchet, P. et Chardenet, P. (dir.) (2011). Guide pour la recherche en didactique des langues et des cultures. Paris : AUF / Editions des archives contemporaines.

Blanchet, P. (2010). Pourquoi s'interroger sur les influences et les enjeux des contextes plurilingues sur les textes et les discours? Éléments pour une théorie de la pluralité linguistique. Dans P. Blanchet, M. Kebbas et Y. Attika Y. (dir.). Influences et enjeux des contextes plurilingues sur les textes et les discours (p. 193-200). Limoges: LambertLucas.

Blumenberg, H. (2005 [2001]). La raison du mythe. Paris : Gallimard.

Bourriaud, N. (2009). Radicant, Pour une esthétique de la globalisation. Paris : Denoël.

Campbell, C. (1987). The Romantic Ethic and the Spirit of Modern Consumerism. Oxford: Basil Blackwell.

Casanova, P. (1999). La république mondiale des Lettres. Paris : Editions du Seuil.

Castellotti, V. et Moore, D. (2008). Contextualisation et universalisme. Quelle didactique des langues pour le $\mathrm{XXI}^{\mathrm{e}}$ siècle ? Dans P. Blanchet, D. Moore et S. Asselah Rahal (dir.), Perspectives pour une didactique des langues contextualisées (p. 183-203). Paris: Éditions des archives contemporaines.

Castellotti, V. et Moore, D. (2011). Dessins d'enfants, recherche qualitative, interprétation. Des poly-textes pour l'étude des imaginaires du plurilinguisme. Dans P. Blanchet et P. Chardenet (dir.), Guide pour la recherche en didactique des langues et des cultures (p. 118-132). Paris : Editions des archives contemporaines.

Cordonier, N. (1996). Victor Segalen, L'expérience de l'œuvre. Paris : Honoré Champion.

De Robillard, D. (2008). Perspectives alterlinguistiques. Paris : 1'Harmattan. 
Deschoux, C.-A. et Zurbriggen, E. (dir.). (2011). 1,2,3 l'école. L'imagier plurilingue, avec CD et cartes en 17 langues. Porrentruy : Editions Migrilude.

Dufour, D.-R. (2007). Le divin marché, La révolution culturelle libérale. Paris : Denoël.

Dufour, D.-R. (2011). L'individu qui vient... après le libéralisme. Paris : Denoël.

Florey, S. (2006). Personnages en quête d'eux-mêmes (Marie NDiaye). L'identité en question ; Versants, Revue suisse des littératures romanes, 52, 31-51.

Glissant, E. (1996). Introduction à une poétique du divers. Paris : Gallimard.

Kaufmann, J.-C. (2004). L'invention de soi. Paris : Armand Colin.

Habermas, J. (1981). La modernité : un projet inachevé. Critique, 413, 950-969.

Hargreaves, A. (1994). Changing Teachers, Changing Times, Teachers' work and culture in the postmodern age. New York: Teachers College.

Kuhn, T. S. (1992, [1962]), La structure des révolutions scientifiques. Paris : Flammarion.

Latour, B. (1991). Nous n'avons jamais été modernes : Essai d'anthropologie symétrique. Paris, La Découverte.

Lipovetsky, G. (1989 [1983]). L'ère du vide. Essais sur l'individualisme contemporain. Paris : Gallimard.

Lipovetsky, G. et Charles, S. (2004), Les temps hypermodernes. Paris : Grasset.

Lyotard, J.-F. (1979). La condition postmoderne. Paris : Minuit.

Lyotard, J.-F. (1982). Réponse à la question : qu'est-ce que le postmoderne ? Critique, 419, 357-367.

Meschonnic, H. (2009). Pour sortir du postmodernisme. Paris : Klincksieck.

Molinié, M. (Dir). (2009). Le dessin réflexif. Elément pour une herméneutique du sujet plurilingue. Amiens : CRTF-Encrages, Belles Lettres.

Morin, E. (2008 [1991]). Francité et universalité. Synergies Monde, 5, 165-170. En ligne : http://ressources-cla.univ-fcomte.fr/gerflint/Monde5/morin.pdf

Ndiaye, M. (2001). Rosie Carpe. Paris : Minuit.

Rorty, R. (1993 [1989]). Contingence, ironie et solidarité. Paris : Armand Colin.

Théofilakis, E. (1985). Les petits récits de chrysalide. Entretien avec J.-F. Lyotard. Modernes et après. Les immatériaux. Paris : Autrement. 